\title{
BIRDS OF THE KARNAPHULI RIVER DELTA AND ADJACENT AREAS IN CHITTAGONG, BANGLADESH
}

\author{
M.F. Ahsan ${ }^{1}$ and M.A. Hannan ${ }^{2}$ \\ ${ }^{1}$ Professor, Department of Zoology, University of Chittagong, Chittagong 4331, Bangladesh \\ ${ }^{2}$ Corresponding author; clo Dr. Md. Anwarul Islam, Professor, Department of Zoology, University of Dhaka, Dhaka 1000, Bangladesh \\ E-mail:mahannan74@yahoo.com
}

\begin{abstract}
A study of the birds of the Karnaphuli River Delta and adjacent areas in Chittagong, Bangladesh, was carried out by direct field observations between July 1999 and June 2000. Status and distribution of the birds were assessed, habitats and some other aspects were also studied. A total of 141 species of birds belonging to 12 orders, 41 families and 98 genera were recorded. Out of 141 species of birds 61 species (43\%) were passerines of which $45(74 \%)$ were resident and $16(26 \%)$ were migratory. Among the 80 non-passerine species, $59(74 \%)$ were resident and 21 (26\%) were migratory. During the study period one species, Brown Rock Chat (Cercomela fusca), was recorded for the first time in Bangladesh .
\end{abstract}

\section{Keywords \\ Birds, Karnaphuli River Delta, Chittagong, Bangladesh}

\section{Introduction}

The aim of the present study was to list the birds found in the study area and to assess their distribution, status and habitats. Bates (1935) recorded some birds from Chittagong. Later on Simmons (1948) listed some bird species for Chittagong. Khanom (1999) registered 92 species of birds from Chittagong University Campus (CUC) which is about $30 \mathrm{~km}$ north of the present study area. The CUC being mainly a hilly area is different from the present place consisting of hills, plain lands and coastal areas. With the establishment of large-scale industries like Chittagong Export Processing Zone (CEPZ), Korean Export Processing Zone (KEPZ), Karnaphuli Fertilizer Company (KAFCO) and Airport among others near the study area, there must have been changes in the faunal composition. However, we hope that the present list will help future workers to have a better idea of the possible changes in the avifaunal composition.

\section{Study Area}

The Karnaphuli River Delta and adjacent areas are the most scenic parts of the Chittagong District with hills and valleys, plain lands, homestead vegetations, sand dunes, sea beach, mangrove forest patches and tidal flats. It includes an area of about $48.88 \mathrm{~km}^{2}$ and lies between $22^{\circ} 12^{\prime} 30^{\prime \prime}$ and $22^{\circ} 20^{\prime} \mathrm{N}$ and between $91^{\circ} 45^{\prime}$ and $91^{\circ} 53^{\prime} \mathrm{E}$ (Fig. 1). The north side of the study area is bounded by Bandar Thana and Char Lakshya Union of Karnaphuli Thana. The south side is surrounded by the Bay of Bengal and Barasat Union of Anwara Thana. The east side is bounded by Jiri and Kasiais Unions of Patiya Thana and Chaturi Union of Anwara Thana. The west side is surounded by the Bay of Bengal.

The naturally grown trees and herbs in this area are Fig (Ficus spp.), Keora (Sonneratia apetala), Goran (Ceriops roxburghiana), Tamarind (Tamarindus indicus), Black berry (Syzygium cumini), Grass (Cynodon dactylon), Reed Chamorro (Phragmites karka), Water hyacinth (Eichhornia crassipes), Gota kola (Centella asiatica), etc. The major timber-yielding species are False Lebbeck (Albizzia spp.), Mehagini (Swietenia mahagoni), Teak (Tectona grandis), etc. Cultivated plants like Mango (Mangifera indica), Jack fruit (Artocarpus heterophyllus), Banana (Musa paradisiaca), Litchi (Litchi chinensis), Guava (Psidium guajava), Coconut (Cocos nucifera), Beetle nut (Areca catechu), etc; agricultural corps like Paddy (Oryza sativa), Potato (Solanum tuberosum), Arum (Alocasia esclenta), Bean (Dolichos lablab), Cucumber (Cucumis sativus), Brinjal (Solanum melongena), Tomato (Lycopersicon lycopersicum), etc are grown. In addition, some of the areas supports monocultures (mainly on the dam and roadside) like Australian Pine (Casuarina equisetifolia), Indian Rosewood (Dalbergia sissoo), Ipil ipil (Leucaena latisiliqua), Asoka (Polyalthia longifolia) and Eucalyptus (Eucalyptus citriodora) planted under social forestry scheme. These vegetations around provide shelter, food and nesting facilities for many species of

Manuscript received 28 August 2001; $\quad$ Revised manuscript received 10 July 2002; $\quad$ Accepted for publication 10 August 2002 


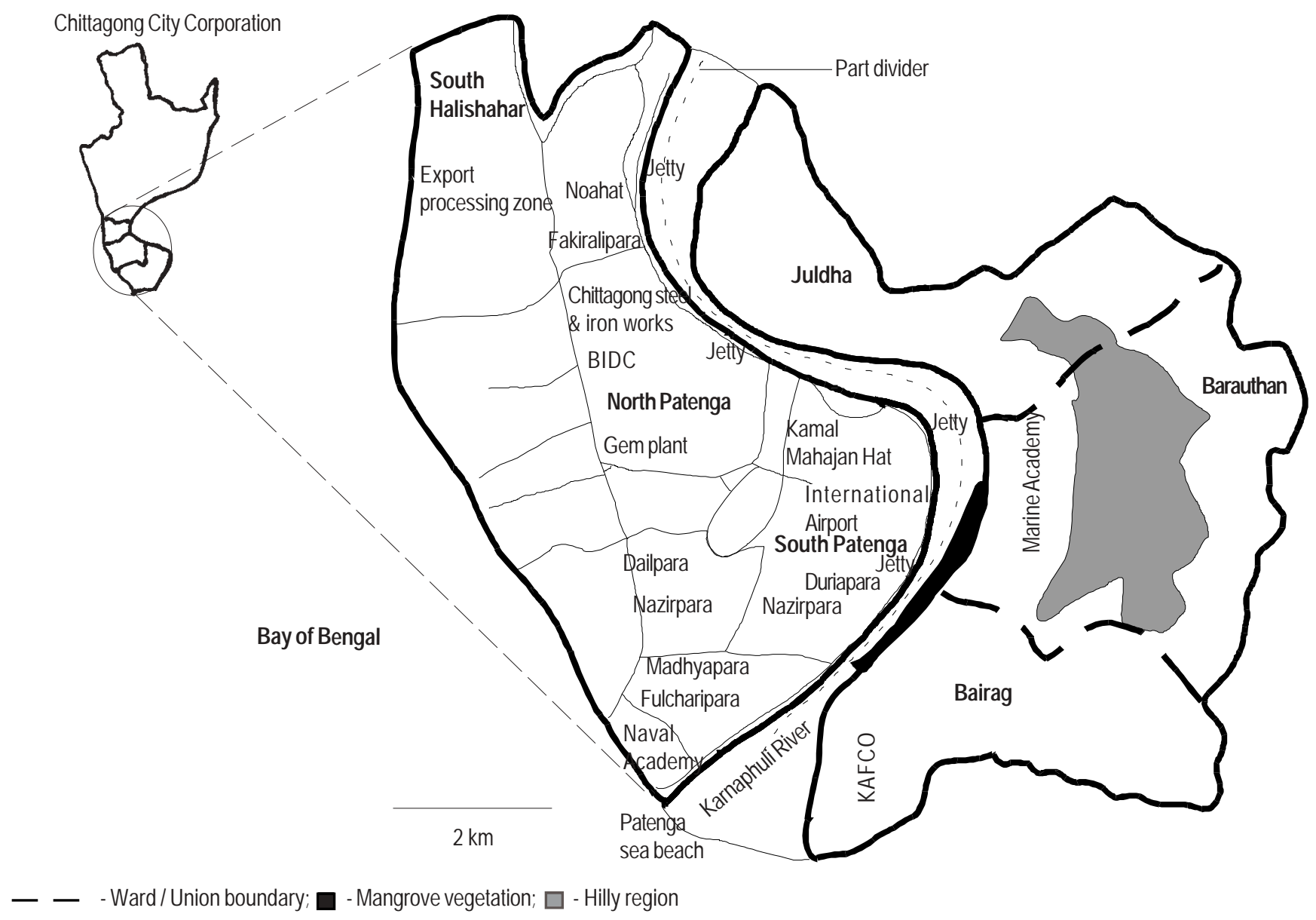

Figure 1. Map of the study area

birds.

\section{Methods}

A year round survey of the birds of the Karnaphuli River Delta and adjacent areas in Chittagong was carried out from July 1999 to June 2000. Birds were observed once a week and in case of missing the schedule it was done the next day. A working day was divided into two parts, viz., morning (0600 to 1100hr) and afternoon (1400 to $1700 \mathrm{hr}$ ). A total of 424 hours in 53 days were spent in the field.

Existing roads, bridle paths, embankments, cattle trails, hill trails, agricultural fields, shore lines, canal and pond banks, etc., were used to cover the study area. The trail length varied from $500 \mathrm{~m}$ to $1000 \mathrm{~m}$ and the visibility of these trails was $50 \mathrm{~m}$ to $100 \mathrm{~m}$ width on both sides. All types of habitats were visited monthly. Maximum field visits were carried on foot but sometimes vehicles were also used to cover long distance of the study area.
Birds were observed either by a pair of binoculars or by naked eyes depending on the distance of the object. Long notes were taken on whether the observed birds were singing, feeding or flying over. For identification, external morphology and other modes i.e., colour, size, shape, flight, walk, habitat, call, and sitting postures were considered; followed by the use of Ali (1996), Ali and Ripley (1983) and/or Sonobe and Usui (1993) for confirmation. A camera with $42-75 \mathrm{~mm}$ zoom and a $300 \mathrm{~mm}$ tele-zoom lens was used for photography.

Status of the recorded birds was assessed on the basis of standard methods followed by Khan (1980). The status were:

Very Common (VC) - bird species, which were recorded on 80$100 \%$ of the field visits;

Common (C) - species that were observed on $50-79 \%$ of the field visits;

Fairly Common (FC) - species that are found on $20-49 \%$ of the field visits; 
Few or Rare (R) - species that were found in less than $19 \%$ of the visits.

The status of birds was strictly followed by calculations, in terms of percentage of occurrence, although, earlier workers (Khan, 1980,1982) determined these categories only based on assumptions and experiences (M.A. Reza Khan, pers. comm.). Besides, resident and migratory species were recognised with the help of old records (e.g. Harvey 1990; Khan, 1982) and / or present observations.

Resident - species that are found round the year and some of which were seen to make nests;

Migratory - species recorded at a certain time of the year.

Three canopies (levels) of vegetation could be recognised in the study area:

1. Lower canopy: maximum height of about $4 \mathrm{~m}$ from the ground, and included bushes, shrubs, small trees and lower parts of big trees,

2. Middle canopy: $4-10 \mathrm{~m}$ above the ground and

3. Upper canopy: above $10 \mathrm{~m}$ high.

The birds have been listed according to the taxonomic classification of Inskipp et al., 1996.

\section{Results and Discussion}

During the study period a total of 141 species of birds (i.e., $21 \%$ of country's total species 683) were recorded. Recorded species belong to 12 orders, 41 families and 98 genera, which comprised $80(57 \%)$ non-passerine and $61(43 \%)$ passerine. Out of 141 species, 104 (74\%) were resident and $37(26 \%)$ were migratory. Of the 80 non-passerine species, 59 (74\%) were resident and 21 (26\%) were migratory. On the other hand, out of 61 passerine species, $45(74 \%)$ were resident and $16(26 \%)$ species were migratory.

Of the total (141) recorded species, $8(6 \%)$ were found to be very common and common, 44 (31\%) were fairly common and 81 (57\%) species were few or rare. It is interesting to mention here that the number of very common and common species were same in this study and the reason for that is inexplicable.

Sand Martin (Riparia riparia) is a migratory species (IUCN Bangladesh, 2000), local winter visitor and passage migrant (Harvey, 1990), and resident (Khan, 1982). This species was observed round the year in the present study area. So, it might be considered as a resident bird of Bangladesh (Khan, 1982).

Many bird species use more than one habitat in the study area. 95 species (67\%) use the hilly area and $57(40 \%)$ use the aquatic habitats (Table 1). Hilly area supports a higher number of species as the area has a comparatively better vegetation cover and is less disturbed than other habitats. The reason why aquatic habitats support few species is inexplicable.

Among the three canopies, individually lower canopy supported highest number of bird species, (25\%) alone and (30\%) of species were found in all the three canopies. Out of 141 species of birds, 14 species are threatened as per IUCN, Bangladesh (IUCN Bangladesh, 2000). Of these 14 species, Pallas' Fish Eagle (Haliaeetus leucoryphus) is Critically Endangered (Locally) and Vulnerable (Globally), Black-bellied Tern (Sterna acuticauda) Endangered (Locally) and Vulnerable (Globally) and Brownwinged Kingfisher (Halcyon amauroptera) is under Lower Risk and the rest 11 are Data Deficient. The status of these 14 species in the area of the present study are: Sand Martin (Riparia riparia) is fairly common and the rest Yellow-fronted Pied Woodpecker (Dendrocopos mahrattensis), Brown-winged Kingfisher (Halcyon amauroptera), Black-bellied Tern (Sterna acuticauda), Pallas's Fish Eagle (Haliaetus leucoryphus), Bar-tailed CuckooDove (Macropygia unchall), White-eyed Buzzard Eagle (Butastur teesa), Black Ibis (Pseudibis papillosa), Golden Oriole (Oriolus oriolus), Crow-billed Drongo (Dicrurus annectans), Ashy Wren-warbler (Prinia socialis), Jungle Wren-warbler (Prinia sylvatica), Straited Marsh Warbler (Megalurus palustris), Streaked Weaver Bird (Ploceus manyar) are rare. Fourteen (10\%) bird species were restricted only to the western part whereas $21(15 \%)$ were restricted to the eastern part while 107 (75\%) species were recorded from both parts (Table 1).

Brown Rock Chat (Cercomela fusca, Blyth 1851) was recorded during October (1999), January, February and April (2000) in the study area, and it is probably the first record of the species in Bangladesh. None of the earlier authors (Rashid, 1967; Husain, 1979; Khan, 1982; Sarker \& Sarker, 1988; Harvey, 1990; Thompson et al., 1993) have reported the Brown Rock Chat from Bangladesh. The range of occurrence months of this species was October to April. So, it is considered as a migratory bird in this present study. Ali (1996) gave the geographical distribution of the Brown Rock Chat as resident in Pakistan and North West India to the Narmada River, Uttar Pradesh, and Bihar to West Bengal.

\section{References}

Ali, S. (1996). The Book of Indian Birds $\left(12^{\text {th }}\right.$ revised and centenary edition). Oxford University Press, New Delhi

Ali, S. and S.D. Ripley (1983). A Pictorial Guides to the Birds of the Indian Subcontinent. Oxford University Press, New Delhi.

Bates, R.S.P. (1935). Some birds of Chittagong. Journal of the Bombay Natural History Society 38: 158-162.

Haque, M.Z. (1991). Zila atlas of Bangladesh, Chittagong and Sylhet Division. Volume-2. Geodesec, Consultants and Services Ltd., Dhaka. Harvey, W.G. (1990). Birds in Bangladesh. University Press Limited, Dhaka.

Husain, K.Z. (1979). Birds of Bangladesh. Department of Films and Publications, Dhaka.

Inskipp, T., N. Lindsely and W. Duckworth (1996). An Annotated Checklist of the Oriental Region. Oriental Bird Club, Berdfordshire, 
Table 1. Systematic list of the recorded species of birds in the study area with their habitats, ecological grouping, status and country distribution.

\begin{tabular}{|c|c|c|c|c|c|c|c|c|}
\hline \multirow[t]{2}{*}{ Scientific name } & \multirow[t]{2}{*}{ Common name } & \multirow[t]{2}{*}{ Local name } & \multirow[t]{2}{*}{ Habitat } & \multicolumn{3}{|c|}{ EG } & \multirow[t]{2}{*}{ 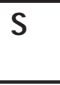 } & \multirow[t]{2}{*}{ D } \\
\hline & & & & UC & MC & LC & & \\
\hline \multicolumn{9}{|l|}{ Anatidae } \\
\hline Tadorna ferruginea (Pallas ,1764) * & Brahminy Shelduck ** & Chokhachokhi/Chokha & 1,2 & & & + & $\mathrm{R}$ & W \\
\hline \multicolumn{9}{|l|}{ Picidae } \\
\hline Jynx torquilla (Linnaeus, 1758) * & Eurasian Wryneck *** & Metho Kaththokra & $5,6,7$ & + & & & $\mathrm{R}$ & W \\
\hline Celeus brachyurus (Vieillot ,1818) & Rufous Woodpcker & Lalchey Kaththokra & $4,5,6$ & + & + & & $\mathrm{R}$ & W \\
\hline Picus xanthopygaeus (J.E. Gray \& & Little Scaly-bellied Green & Sabujey Kaththokra & $2,4,5,6,7$ & + & + & & $\mathrm{R}$ & W \\
\hline & & & & & & & & \\
\hline Dinopium benghalense (Linnaeus, 1758) & Lesser Golden-backed Woodpecker & r Kaththokra & $2,5,6,7$ & + & + & + & $\mathrm{FC}$ & W \\
\hline Dinopium javanense (Ljungh, 1797) & $\begin{array}{l}\text { Common Golden-backed } \\
\text { Woodpecker }\end{array}$ & & $5,6,7$ & + & & & $\mathrm{R}$ & MEF, SB \\
\hline Dendrocopos macei (Vieillot ,1818) & Fulvous-breasted Pied Woodpecker & r Pakra Kaththokra & $4,6,7$ & & + & & $\mathrm{R}$ & W \\
\hline Dendrocopos mahrattensis (Latham, 1801) & Yellow-fronted Pied Woodpecker & Pakra Kaththokra & $2,5,6,7$ & + & + & & $\mathrm{R}$ & W \\
\hline \multicolumn{9}{|l|}{ Capitonidae } \\
\hline Megalaima lineata (Vieillot, 1816) & Lineated Barbet & Gurkhod, Beghbou & $3,5,6,7$ & + & + & & $\mathrm{R}$ & W \\
\hline \multicolumn{9}{|l|}{ Upupidae } \\
\hline Upupa epops Linnaeus, 1758 & Common Hoopoe & Hudhud, Slaiman Pakhi & $2,3,6$ & & + & + & $\mathrm{R}$ & W \\
\hline \multicolumn{9}{|l|}{ Alcedinidae } \\
\hline Alcedo atthis (Linnaeus, 1758) & Small Blue Kingfisher & Choto Maachranga & $1,2,3,4,5,6,7$ & + & + & + & $\mathrm{C}$ & W \\
\hline Halcyon amauroptera Pearson, 1841 & Brown-winged Kingfisher *** & & 1,2 & & + & & $\mathrm{R}$ & SB \\
\hline Halcyon capensis (Linnaeus, 1766) & Stork-billed Kingfisher $* \star \star$ & Megh-hao & 2,7 & & + & + & $\mathrm{R}$ & W \\
\hline Halcyon smyrnensis (Linnaeus, 1758) & White-breasted Kingfisher & Maachranga & $1,2,3,4,5,6$ & + & + & + & FC & W \\
\hline Halcyon pileata (Boddaert, 1783) & Black-capped Kingfisher ** & & 2 & + & + & & $\mathrm{R}$ & $\begin{array}{l}\text { Coast } \\
\text { including } \\
\text { StM }\end{array}$ \\
\hline Ceryle rudis (Linnaeus, 1758) & Lesser Pied Kingfisher ** & Pakra Maachranga & 1,2 & & + & + & $\mathrm{R}$ & W \\
\hline \multicolumn{9}{|l|}{ Meropidae } \\
\hline Merops leschenaulti Vieillot, 1817 & Chestnut-headed Bee-eater *** & Lal Matha Suichora & $2,3,5,6$ & + & + & + & $\mathrm{FC}$ & Hills \\
\hline Merops philippinus Linnaeus, 1766 & Blue-tailed Bee-eater ${ }^{\star \star \star}$ & Nil Lej Suichora & $3,5,6$ & + & + & + & FC & W \\
\hline Merops orientalis Latham, 1801 & Small Bee-eater & Suichora, Banspati & 5 & + & + & + & $\mathrm{R}$ & W \\
\hline \multicolumn{9}{|l|}{ Cuculidae } \\
\hline Clamator jacobinus (Boddaert, 1783) & Pied Crested Cuckoo *** & Chokhgelo Pakhi & 5,6 & & + & & $\mathrm{R}$ & W \\
\hline Hierococcyx varius (Vahl, 1797) & Brainfever Bird & Kalo Kokil, kuli & 5,6 & + & + & & $\mathrm{R}$ & W \\
\hline Cuculus micropterus Gould, 1838 & Indian Cuckoo *** & & 5,6 & + & + & & $\mathrm{R}$ & W \\
\hline Eudynamus scolopacea (Linnaeus, 1758) & Asian Koel & Kanakuka & $4,5,6,7$ & + & + & & $\mathrm{R}$ & W \\
\hline Centropus sinensis (Stephens, 1815) & Greater Coucal & & $5,6,7$ & & + & + & $\mathrm{R}$ & W \\
\hline Centropus bengalensis (Gmelin, 1788) & Lesser Coucal & & $4,6,7$ & & + & + & $\mathrm{R}$ & Hill forest \\
\hline \multicolumn{9}{|l|}{ Psittacidae } \\
\hline Psittacula krameri (Scopoli, 1769) & Rose-ringed Parakeet & Tia & $3,4,5,6,7$ & + & + & + & $\mathrm{FC}$ & W \\
\hline \multicolumn{9}{|l|}{ Apodidae } \\
\hline Apus affinis (J.E. Gray, 1830) & House Swift & Ababil & $2,3,4,5,6$ & + & + & + & $\mathrm{FC}$ & W \\
\hline Cypsiurus balasiensis (J.E. Grey, 1829) & Asian Palm-Swift & Nakkati & $1,2,3,4,5,6,7$ & + & + & + & $\mathrm{C}$ & W \\
\hline \multicolumn{9}{|l|}{ Tytonidae } \\
\hline Tyto alba (Scopoli, 1769) & Barn Owl & Laxmi Pencha & $5,6,7$ & + & + & & $\mathrm{R}$ & W \\
\hline
\end{tabular}




\begin{tabular}{|c|c|c|c|c|c|c|c|c|}
\hline \multirow[t]{2}{*}{ Scientific name } & \multirow[t]{2}{*}{ Common name } & \multirow[t]{2}{*}{ Local name } & \multirow[t]{2}{*}{ Habitat } & \multicolumn{3}{|c|}{ EG } & \multirow[t]{2}{*}{$\mathrm{S}$} & \multirow[t]{2}{*}{ D } \\
\hline & & & & UC & MC & $\mathrm{LC}$ & & \\
\hline \multicolumn{9}{|l|}{ Strigidae } \\
\hline Athene brama (Temminck, 1821) & Spotted Owlet & Kuruley Pencha & 5,6 & + & + & & $\mathrm{R}$ & W \\
\hline \multicolumn{9}{|l|}{ Columbidae } \\
\hline Treron phoenicoptera (Latham, 1790) & Yellow-legged Green-Pigeon *** & Horial, Botkol & $3,5,6,7$ & + & & & $\mathrm{R}$ & W \\
\hline Columba livia Gmelin, 1789 & Blue Rock Pigeon & Jalali Kobutor, Kapot & $1,2,3,4,5,6,7$ & + & + & + & C & W \\
\hline Macropygia unchall (Wagler, 1827) & Barred Cuckoo-Dove *** & & $3,4,5,6$ & & + & + & $\mathrm{R}$ & $?$ \\
\hline Streptopelia orientalis (Latham, 1790) * & Oriental Turtle-Dove & Ghughu & $3,5,6,7$ & + & + & + & $\mathrm{FC}$ & MEF, SB \\
\hline Streptopelia decaocto (Frivaldszky, 1838) & Eurasian Collared-Dove & Raj Ghughu & $2,3,4,5,6,7$ & + & + & + & $\mathrm{FC}$ & W \\
\hline Streptopelia tranquebarica (Hermann, 1804) & Red Collared-Dove & Lal Ghughu, Jongla & $2,3,5,6$ & + & + & + & $\mathrm{R}$ & W \\
\hline Streptopelia chinensis (Scopoli, 1786) & Spotted Dove & Tila Ghughu & $2,3,4,5,6,7$ & + & + & + & C & W \\
\hline \multicolumn{9}{|l|}{ Rallidae } \\
\hline Porzana fusca (Linnaeus, 1766) & Ruddy-breasted Crake ** & Ranga Crake & 1 & & & + & $\mathrm{R}$ & SE, Hoars \\
\hline Amaurornis phoenicurus (Pennant, 1769) & White-breasted Waterhen & Dahuk & $1,2,3,5$ & & & + & $\mathrm{FC}$ & W \\
\hline \multicolumn{9}{|l|}{ Jacanidae } \\
\hline Metopidius indicus (Latham, 1790) & Bronze-winged Jacana ** & Jolpipi, Pipi & 1,3 & & & + & $\mathrm{FC}$ & W \\
\hline \multicolumn{9}{|l|}{ Charadriidae } \\
\hline Vanellus indicus (Boddaert, 1783) & Red-wattled Lapwing & Lal-lotika Hot-ti-ti & $1,2,3$ & & & + & $\mathrm{FC}$ & W \\
\hline Vanellus malabaricus (Boddaert, 1783) & Yellow-wattled Lapwing ** & Hot-ti-ti & 2,3 & & & + & $\mathrm{FC}$ & W \\
\hline Charadrius hiaticula Linnaeus, 1758 * & Common Ringed Plover & & $1,2,3$ & & & + & $\mathrm{R}$ & $\begin{array}{l}\text { Cox's } \\
\text { Bazar in } \\
\text { MF, SF }\end{array}$ \\
\hline Charadrius dubius Scopoli, 1786 * & Little Ringed Plover & Choto Jiria & 1,2 & & & + & $\mathrm{FC}$ & W \\
\hline Charadrius alexandrinus Linnaeus, 1758 * & Kentish Plover & Jiria & 1,2 & & & + & $\mathrm{FC}$ & W \\
\hline Charadrius mongolus Pallas, 1776 * & Lesser Sand Plover ** & & $1,2,3$ & & & + & $\mathrm{FC}$ & W \\
\hline Pluvialis fulva (Gmelin, 1789) * & Pacific Golden Plover ** & Batan & $1,2,3$ & & & + & $\mathrm{R}$ & W \\
\hline \multicolumn{9}{|l|}{ Scolopacidae } \\
\hline Tringa totanus (Linnaeus, 1758)* & Common Redshank & Lalpa Pi-0o & 2 & & & + & $\mathrm{R}$ & W \\
\hline Tringa stagnatilis (Bechstein, 1803) * & Marsh Sandpiper & Jalar Chapakhi & 1,2 & & & + & $\mathrm{R}$ & W \\
\hline Tringa nebularia (Gunner, 1767)* & Common Greenshank & & 1,2 & & & + & $\mathrm{R}$ & \\
\hline Actitis hypoleucos Linnaeus, 1758 * & Common Sandpiper & Chapakhi & $1,2,3$ & & & + & $\mathrm{FC}$ & W \\
\hline Gallinago stenura (Bonaparte, 1830) * & Pintail Snipe & Kada-khocha/Chaga & $1,2,3,4$ & & & + & $\mathrm{FC}$ & W \\
\hline Gallinago gallinago (Linnaeus, 1758) * & Common Snipe & Kada-khocha/Chaga & $2,3,4$ & & & + & $\mathrm{R}$ & W \\
\hline Calidris minuta (Leisler, 1812) * & Little Stint & & 1,2 & & & + & $\mathrm{FC}$ & W \\
\hline Calidris temminckii (Leisler, 1812) * & Temminck's Stint & & $1,2,3$ & & & + & $\mathrm{R}$ & W \\
\hline \multicolumn{9}{|l|}{ Laridae } \\
\hline Larus brunnicephalus Jerdon, 1840 * & Brown-headed Gull & Gonga Koitar & 1,2 & & & + & $\mathrm{R}$ & W \\
\hline Larus ridibundus Linnaeus, 1766* & Black-headed Gull & Gonga Koitar & 1,2 & & & + & $\mathrm{FC}$ & $\begin{array}{l}\text { Coasts, } \\
\text { Padma }\end{array}$ \\
\hline Chlidonias hybridus (Pallas, 1811) * & Whiskered Tern & & 1,2 & & & + & $\mathrm{R}$ & W \\
\hline Sterna aurantia J.E. Gray, 1831 & River Tern & Maach Khaikka & 1,2 & & & + & $\mathrm{FC}$ & W \\
\hline Sterna hirundo Linnaeus, 1758 * & Common Tern & Gangchil & 1,2 & & & + & $\mathrm{R}$ & W \\
\hline Sterna acuticauda J.E. Gray, 1831 & Black-bellied Tern & Gangchil & 1,2 & & & + & $\mathrm{R}$ & W \\
\hline Sterna albifrons Pallas, 1764 & Little Tern & Khudey Gangchil & 1,2 & & & + & $\mathrm{FC}$ & W \\
\hline \multicolumn{9}{|l|}{ Accipitridae } \\
\hline Milvus migrans (Boddaert, 1783) & Black Kite & Bhuban Chil & $2,3,4,5,6,7$ & + & + & + & $\mathrm{FC}$ & W \\
\hline Milvus milvus Linnaeus, 1758 * & Red Kite & Bhuban Chil & $1,2,3,4,6,7$ & + & + & + & - & W \\
\hline Haliastur indus (Boddaert, 1783) & Brahminy Kite *** & Shankho Chil, Lal Chil & 5,6 & + & + & & $\mathrm{R}$ & $\begin{array}{l}\text { Forestedge } \\
\text { \& clearings }\end{array}$ \\
\hline Butastur teesa (Franklin, 1832) & White-eyed Buzzard *** & Buj, Eagle & 5,6 & + & & & $\mathrm{R}$ & W \\
\hline
\end{tabular}




\begin{tabular}{|c|c|c|c|c|c|c|c|c|}
\hline \multirow[t]{2}{*}{ Scientific name } & \multirow[t]{2}{*}{ Common name } & \multirow[t]{2}{*}{ Local name } & \multirow[t]{2}{*}{ Habitat } & \multicolumn{3}{|c|}{ EG } & \multirow[t]{2}{*}{$\mathbf{S}$} & \multirow[t]{2}{*}{ D } \\
\hline & & & & UC & $\mathrm{MC}$ & LC & & \\
\hline Haliaeetus leucoryphus (Pallas, 1771) & Pallas's Fish-Eagle & Kura, Bo-wol & 1,5 & + & + & + & $\mathrm{R}$ & W \\
\hline Spilornis cheela (Latham, 1790) & Crested Serpent-Eagle & Tila Baj, Shapkheko Baj & $2,3,5,7$ & + & + & & $\mathrm{R}$ & W \\
\hline \multicolumn{9}{|l|}{ Falconidae } \\
\hline Falco tinnunculus Linnaeus, 1758 * & Common Kestrel ${ }^{\star \star \star}$ & Baj & 5 & + & & & $\mathrm{R}$ & W \\
\hline \multicolumn{9}{|l|}{ Phalacrocoracidae } \\
\hline Phalacrocorax niger (Vieillot, 1817) & Little Cormorant & Paan-kowri/Pani Kaor & $1,2,3,4,6$ & + & + & + & $\mathrm{FC}$ & W \\
\hline \multicolumn{9}{|l|}{ Ardeidae } \\
\hline Ardea cinerea Linnaeus, 1758 & Grey Heron ** & Dushor Bok & 1,2 & & & + & $\mathrm{R}$ & W \\
\hline Casmerodius albus (Linnaeus, 1758) & Large Egret ** & Bara Sada Bok & $1,2,6$ & & & + & $\mathrm{R}$ & W \\
\hline Ardeola grayii (Sykes, 1832) & Indian Pond-Heron & Kani Bok & $1,2,3,4,5,6,7$ & + & + & + & VC & W \\
\hline Bubulcus ibis (Linnaeus, 1758) & Cattle Egret & Go-bok & $1,2,3,4,5,6,7$ & & + & + & VC & \\
\hline Mesophoyx intermedia (Wagler, 1829) & Median Egret ** & Maijla Bok & 1,2 & & & + & $\mathrm{R}$ & W \\
\hline Egretta garzetta (Linnaeus, 1766) & Little Egret & Choto Bok & 1,2 & & + & + & $\mathrm{FC}$ & W \\
\hline Nycticorax nycticorax (Linnaeus, 1758) & Black-crowned Night-Heron ** & Waak, Nishi Bok & 6 & & + & & $\mathrm{FC}$ & W \\
\hline Ixobrychus cinnamomeus (Gmelin, 1789) & Chestnut Bittern *** & Lal Bok & $1,2,3$ & & & + & $\mathrm{FC}$ & W \\
\hline Ixobrychus sinensis (Gmelin, 1789) & Yellow Bittern ** & $\begin{array}{l}\text { Holdey Bok, Korchey } \\
\text { Bok }\end{array}$ & 1,3 & & & + & $\mathrm{R}$ & W \\
\hline \multicolumn{9}{|l|}{ Threskiornithidae } \\
\hline Pseudibis papillosa (Temminck, 1824) & Black Ibis ** & Kalo Duchora & 2 & & & + & $\mathrm{R}$ & W \\
\hline \multicolumn{9}{|l|}{ Ciconiidae } \\
\hline Anastomus oscitans (Boddaert, 1783) & Asain Openbill Stork *** & $\begin{array}{l}\text { Shamuk Bhanga, } \\
\text { Shamuk-Khol }\end{array}$ & 2 & & & + & $\mathrm{R}$ & Coast \\
\hline \multicolumn{9}{|l|}{ Irenidae } \\
\hline Chloropsis cochinchinensis (Gmelin, 1788) & Jerdon's Chloropsis & Sabuj Bulbuli & $4,5,6,7$ & + & & & $\mathrm{R}$ & W \\
\hline Aegithina tiphia (Linnaeus, 1758) & Common lora & Towfik, Fatikjal & $5,6,7$ & & + & & $\mathrm{R}$ & W \\
\hline \multicolumn{9}{|l|}{ Laniidae } \\
\hline Lanius cristatus Linneaus, 1758 * & Brown Shrike & Badami Koshai Pakhi & $2,3,4,5,6,7$ & + & + & + & $\mathrm{FC}$ & MEF \\
\hline \multicolumn{9}{|l|}{ Oriolidae } \\
\hline Oriolus oriolus (Linnaeus, 1758) & Eurasian Golden Oriole *** & Beney Bou & $5,6,7$ & + & & & $\mathrm{R}$ & W \\
\hline Oriolus xanthornus (Linnaeus, 1758) & Black-headed Oriole & Holdey Pakhi & $4,5,6$ & + & + & + & $\mathrm{R}$ & $\mathrm{N}$ \\
\hline \multicolumn{9}{|l|}{ Dicruridae } \\
\hline Dicrurus macrocercus Vieillot, 1817 & Black Drongo & Fingey & $1,2,3,4,5,6,7$ & + & + & + & VC & W \\
\hline Dicrurus leucophaeus Vieillot, 1817 * & Ashy Drongo & Nil Fingey & 3,5 & & + & + & $\mathrm{R}$ & W \\
\hline Dicrurus annectans (Hodgson, 1836) & Crow-billed Drongo & & $3,5,6$ & & + & + & $\mathrm{R}$ & W \\
\hline Dicrurus aeneus Vieillot, 1817 & Bronzed Drongo & Choto Fingey & $3,5,6$ & + & + & + & $\mathrm{FC}$ & MEF \\
\hline \multicolumn{9}{|l|}{ Corvidae } \\
\hline Corvus splendens Vieillot, 1817 & House Crow & Pati Kak, Kawa & $1,2,3,4,5,6,7$ & + & + & + & VC & W \\
\hline Corvus macrorhynchos Wagler, 1827 & Jungle Crow & Dar Kak & $2,4,5,6,7$ & + & + & + & $\mathrm{FC}$ & W \\
\hline Dendrocitta vagabunda (Latham, 1790) & Indian Treepie & Harichacha, Kutum & $4,5,6,7$ & + & + & & FC & W \\
\hline \multicolumn{9}{|l|}{ Campephagidae } \\
\hline Tephrodornis pondicerianus (Gmelin, 1789) & Common Woodshrike & Koshi Pakhi & $2,4,5,6,7$ & + & + & & $\mathrm{R}$ & W \\
\hline Coracina macei (Lesson, 1830) & Large Cuckooshrike & Gudhuka & $5,6,7$ & + & + & & $\mathrm{R}$ & W \\
\hline Pericrocotus cinnamomeus (Linnaeus, 1766) & Small Minivet & Sat Saili & $4,5,6,7$ & + & + & & $\mathrm{R}$ & W \\
\hline
\end{tabular}




\begin{tabular}{|c|c|c|c|c|c|c|c|c|}
\hline \multirow[t]{2}{*}{ Scientific name } & \multirow[t]{2}{*}{ Common name } & \multirow[t]{2}{*}{ Local name } & \multirow[t]{2}{*}{ Habitat } & \multicolumn{3}{|c|}{ EG } & \multirow[t]{2}{*}{$\mathrm{S}$} & \multirow[t]{2}{*}{ D } \\
\hline & & & & UC & MC & LC & & \\
\hline \multicolumn{9}{|l|}{ Muscicapidae } \\
\hline Rhipidura albicollis (Vieillot, 1818) & White-throated Fantail-Flycatcher & Lejnachani & $2,4,5,6,7$ & + & + & & $\mathrm{FC}$ & \\
\hline Ficedula parva (Bechstein, 1792) * & Red-throated Flycatcher & Lalbook Chotok & $2,4,5,6,7$ & + & + & + & $\mathrm{R}$ & W \\
\hline Culicicapa ceylonensis (Swainson, 1820) & Grey-headed Flycatcher & Footfuti & $2,4,5,6,7$ & + & + & + & $\mathrm{R}$ & W \\
\hline Copsychus saularis (Linnaeus, 1758) & Oriental Magpie-Robin & Doel, Doinachani & $1,2,3,4,5,6,7$ & + & + & + & VC & W \\
\hline Copsychus malabaricus (Scopoli, 1786) & 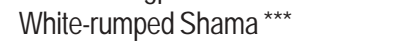 & Shama & 5,6 & & + & + & $\mathrm{R}$ & \\
\hline Saxicola torquata (Linnaeus, 1766) * & Common Stonechat & & 2,3 & & + & + & $\mathrm{R}$ & $\begin{array}{l}\text { Forest, } \\
\text { woods }\end{array}$ \\
\hline Cercomela fusca (Blyth, 1851) * & Indian Chat & & 2,4 & & & + & $\mathrm{R}$ & W \\
\hline Prinia socialis Sykes,1832 & Ashy Prinia & Prinia & $1,2,5,6,7$ & & + & & $\mathrm{R}$ & W \\
\hline Prinia sylvatica Jerdon, 1840 & Jungle Prinia & & $3,5,6$ & & + & + & $\mathrm{R}$ & $\mathrm{NE}$ \\
\hline Locustella certhiola (Pallas, 1811) * & Rusty-rumped Grasshopper-Warbler & & $2,3,4,5,6,7$ & & + & + & $\mathrm{R}$ & $?$ \\
\hline Megalurus palustris Horsfield, 1821 & Straited Marsh-Warbler & & $1,2,3,4,5,6$ & & + & + & $\mathrm{R}$ & Hills, hoars \\
\hline Orthotomus sutorius (Pennant, 1769) & Common Tailor Bird & Tuntuni & $1,2,3,4,5,6,7$ & + & + & + & $\mathrm{C}$ & W \\
\hline Phylloscopus affinis (Tickell, 1833) * & Tickell's Warbler *** & & $2,5,6$ & & + & + & $\mathrm{R}$ & W \\
\hline Macronous gularis (Horsfield, 1822) & Yellow-breasted Babbler & & 5,6 & & + & + & $\mathrm{R}$ & W \\
\hline Turdoides striatus (Dumont, 1823) & Jungle Babbler & Satbhai, Satbhaira & $4,5,6,7$ & & + & + & FC & W \\
\hline Alcippe poioicephala (Jerdon, 1844) & Quaker Tit-Babbler & & $3,5,6,7$ & & + & + & $\mathrm{R}$ & W \\
\hline \multicolumn{9}{|l|}{ Sturnidae } \\
\hline Sturnus malabaricus (Gmelin, 1789) & Grey-headed Starling & Kath Shalik & $2,3,4,5,6,7$ & + & + & + & $\mathrm{FC}$ & \\
\hline Sturnus contra Linnaeus, 1758 & Asian Pied Starling & Gobrey/Go Shalik & $1,2,3,4,5,6,7$ & + & + & + & VC & W \\
\hline Acridotheres tristis (Linneaus, 1766) & Common Myna & Bhat Shalik & $1,2,3,4,5,6,7$ & + & + & + & VC & W \\
\hline Acridotheres ginginianus (Latham, 1790) & Bank Myna & Gang Shalik & $1,2,3,4,5,6,7$ & & + & + & $\mathrm{R}$ & W \\
\hline Acridotheres fuscus (Wagler, 1827) & Jungle Myna & Jhuti Shalik & $1,2,3,4,5,6,7$ & + & + & + & $\mathrm{C}$ & W \\
\hline \multicolumn{9}{|l|}{ Hirundinidae } \\
\hline Riparia riparia (Linnaeus, 1758) & Sand Martin & Nakkati & $1,2,5,6,7$ & + & + & & FC & W \\
\hline Hirundo rustica Linnaeus, 1758 * & Common Swallow & Ababil & $1,2,3,5,6,7$ & + & + & & FC & W \\
\hline \multicolumn{9}{|l|}{ Paridae } \\
\hline Parus major Linnaeus, 1758 & Great Tit & Tit Pockh & $1,2,3,4,5,6,7$ & + & + & + & FC & W \\
\hline \multicolumn{9}{|l|}{ Pycnonotidae } \\
\hline Pycnonotus jocosus (Linnaeus, 1758) & Red-whiskered Bulbul & Shpahi Bulbuli & $1,2,3,4,5,6,7$ & & + & + & $\mathrm{R}$ & W \\
\hline Pycnonotus cafer (Linnaeus, 1766) & Red-vented Bulbul & Bulbuli & $1,2,3,4,5,6,7$ & + & + & + & C & W \\
\hline \multicolumn{9}{|l|}{ Zosteropidae } \\
\hline Zosterops palpebrosus (Temminck, 1824) & Oriental White-eye *** & Shet-akkhi & 5,7 & & + & & $\mathrm{R}$ & W \\
\hline \multicolumn{9}{|l|}{ Alaudidae } \\
\hline Mirafra assamica Horsfield, 1840 & Bengal Bush-Lark & Bharat Pakhi & $2,3,5,6$ & & + & + & $\mathrm{R}$ & \\
\hline \multicolumn{9}{|l|}{ Dicaeidae } \\
\hline Dicaeum erythrorhynchos (Latham, 1790) & Tickell's Flowerpecker & Fuljhuri & $3,4,5,6,7$ & & + & + & FC & W \\
\hline \multicolumn{9}{|l|}{ Nectariniidae } \\
\hline Nectarinia zeylonica (Linnaeus, 1766) & Purple-rumped Sunbird & Mautushi & $4,5,6,7$ & + & + & & $\mathrm{R}$ & W \\
\hline Nectarinia asiatica (Latham, 1790) & Purple Sunbird & Niltuni, Madhuchushki & $5,6,7$ & + & + & & FC & W \\
\hline Arachnothera longirostra (Latham, 1790) & Little Spiderhunter & & $5,6,7$ & + & + & & $\mathrm{R}$ & $\begin{array}{l}\text { Forests, } \\
\text { woods }\end{array}$ \\
\hline \multicolumn{9}{|l|}{ Motacillidae } \\
\hline Anthus hodgsoni Richmond, 1907 * & Oriental Tree Pipit & Gecho Pipit & $2,3,4,5,6,7$ & + & + & & FC & W \\
\hline Anthus rufulus Vieillot, 1818* & Paddyfield Pipit & Dhani Pipit & $2,3,4,5,6,7$ & + & + & + & $\mathrm{FC}$ & W \\
\hline
\end{tabular}




\begin{tabular}{|c|c|c|c|c|c|c|c|c|}
\hline \multirow[t]{2}{*}{ Scientific name } & \multirow[t]{2}{*}{ Common name } & \multirow[t]{2}{*}{ Local name } & \multirow[t]{2}{*}{ Habitat } & \multicolumn{3}{|c|}{ EG } & \multirow[t]{2}{*}{$\mathbf{S}$} & \multirow[t]{2}{*}{ D } \\
\hline & & & & UC & MC & LC & & \\
\hline Motacilla flava Linneaus, 1758 * & Yellow Wagtail & Halud Khonjan & $1,2,3,4,5,6,7$ & & + & + & $\mathrm{R}$ & W \\
\hline Motacilla citreola Pallas, 1776 * & Citrine Wagtail & Halud Matha Khonjan & $1,2,3,4,5,6,7$ & & + & + & $\mathrm{R}$ & W \\
\hline Motacilla cinerea Tunstall, 1771 * & Grey Wagtail & Khonjan & $2,3,4,5,6,7$ & & & + & $\mathrm{FC}$ & W \\
\hline Motacilla alba Linnaeus, 1758* & White Wagtail & Sada Khonjan & $2,3,4,5,6,7$ & & + & + & FC & W \\
\hline Motacilla maderaspatensis Gmelin, 1789 & Large Pied Wagtail & Pakra Khonjan & 3,6 & & + & & $\mathrm{R}$ & W \\
\hline \multicolumn{9}{|l|}{ Passeridae } \\
\hline Passer domesticus (Linnaeus, 1758) & House Sparrow & Charui & $1,2,3,4,5,6,7$ & + & + & + & VC & W \\
\hline Passer montanus (Linnaeus, 1758) * & Eurasian Tree Sparrow & Gecho Charui & $3,4,5,6,7$ & + & + & + & $\mathrm{FC}$ & SE \\
\hline Ploceus philippinus (Linnaeus, 1766) & Baya Weaver & Babui, Baoi & $2,3,4,5,6,7$ & + & + & + & C & \\
\hline Ploceus manyar (Horsfield, 1821) & Streaked Weaver & Dora Chata Babui & $2,4,5,6$ & + & + & + & $\mathrm{FC}$ & W \\
\hline \multicolumn{9}{|l|}{ Estrildidae } \\
\hline Amandava amandava (Linnaeus, 1758) & 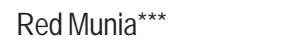 & Lal Munia & 5,7 & & & + & $\mathrm{R}$ & W \\
\hline Lonchura punctulata (Linnaeus, 1758) & Spotted Munia *** & Tila Munia & $1,2,3,5$ & & + & + & $\mathrm{R}$ & W \\
\hline \multicolumn{9}{|l|}{ Fringillidae } \\
\hline Caprodacus erythrinus (Pallas, 1770)* & Common Rosefinch $* \star *$ & Lal Matha Finch & 5 & & + & + & $\mathrm{R}$ & SE \\
\hline
\end{tabular}

Habitats: 1. Aquatic Habitat, 2. Semi-aquatic Habitat, 3. Agricultural Field, 4. Planted Vegetation, 5. Hilly Area, 6. Homestead Vegetation and 7. Industrial Area EG - Ecological grouping: UC - upper canopy, LC - lower canopy and MC - middle canopy; + present; - absent

Status: $\mathrm{VC}$ - very common, $\mathrm{C}$ - common, $\mathrm{FC}$ - fairly common and $\mathrm{R}$ - rare or few

Distribution: W - Wide, N - North, S - South, E - East, C - Central, NE - Northeast, NW - Northwest, SE - Southeast, StM - St. Martin's Island, DF - Deciduous Forest, MEF - Mixed Evergreen Forest; SB - Sundarbans Mangrove Forest; * Indicating migratory species and the rest are resident; ** Species restricted only in the western part; *** - Species restricted only in the eastern part

U.K.

IUCN Bangladesh (2000). Red Book of Threatened Birds of Bangladesh. IUCN, Bangladesh..

Khan, M.A.R. (1982). Wildlife of Bangladesh: A Checklist. Dhaka University Press, Dhaka.

Khan M.A.R. (1980). A comparative account of the avifauna of the sholas and the neighbouring plantation in the Nilgiri. Journal of the Bombay Natural History Society 75(3): 1028-1035.

Khanom, N. (1999). Birds of Chittagong University Campus (with notes on status, distribution and habitat). M.Sc. Thesis. Chittagong University, Chittagong (unpublished).

Rashid, H. (1967). Systematic List of the Birds of East Pakistan. The Asiatic Society of East Pakistan, Dacca.

Sarker, M.S.U. and N.J. Sarker (1988). Wildlife of Bangladesh -- A Systematic List with Status, Distribution and Habitat. The Rico Printers, Dhaka.

Simmons, R.M. (1948). A list of birds observed in Chittagong, East Bengal during 1944 and 1945. Journal of the Bombay Natural History Society 17(3): 637-644.

Sonobe, K. and S. Usui (Editors) (1993). A Field Guide to the Water Birds of Asia. Wild Bird Society of Japan, Tokyo.

Thompson, P. M., W.G. Harvey, D.L. Johnson, D.J. Millin, S.M.A. Rashid, D., A. Scott, C. Stanford and D. Woolner (1993). Recent notable bird records from Bangladesh. Forktail 9: 12-44. 